PREPRINT

AS54

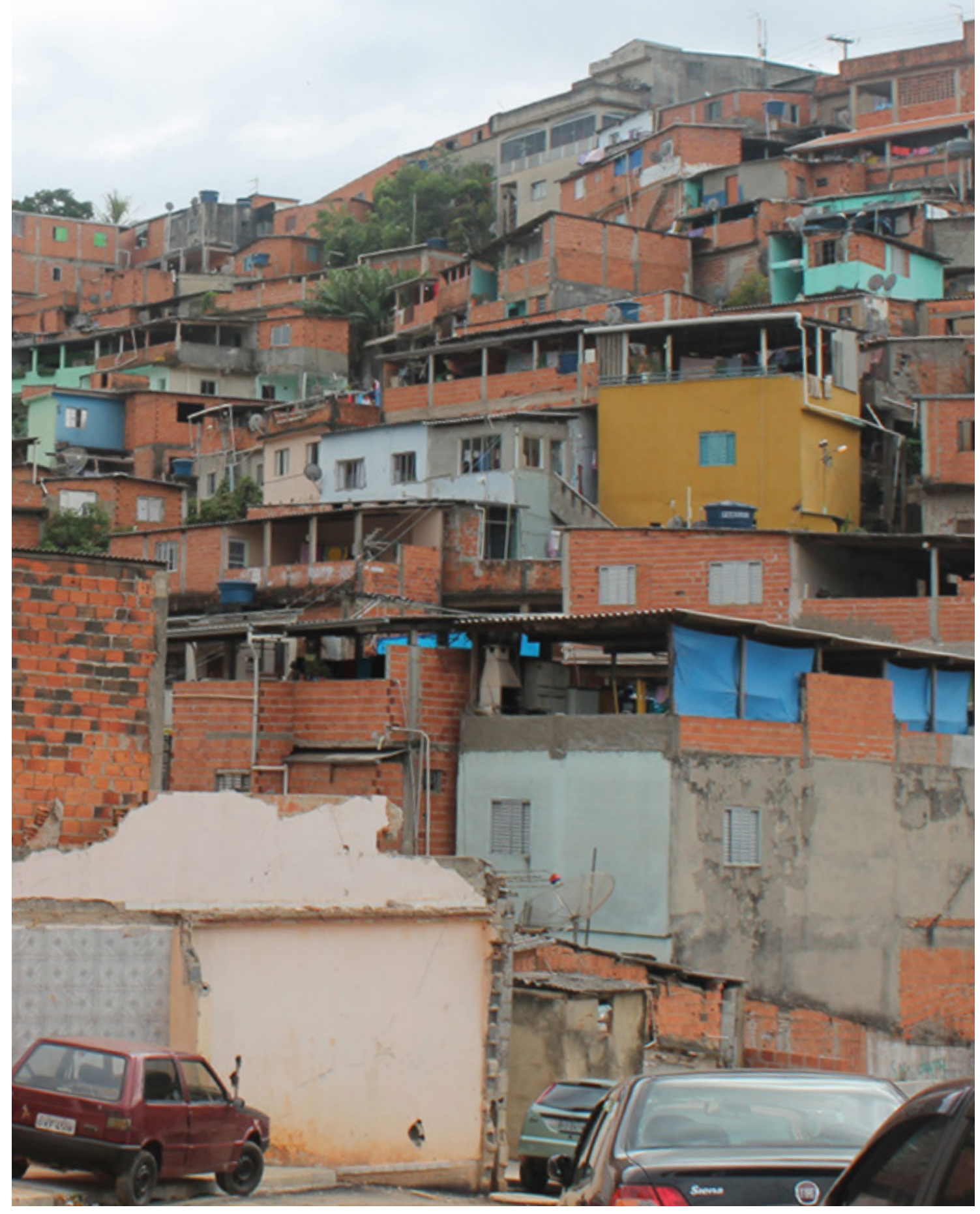

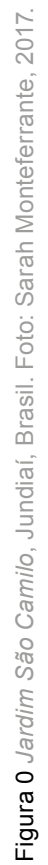



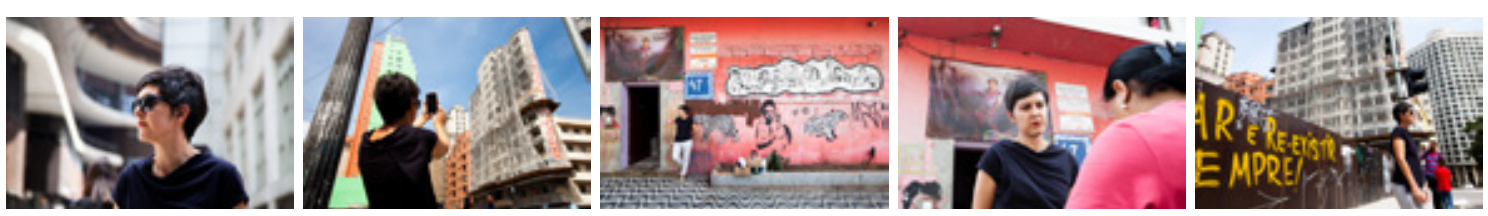

\title{
EL DESARROLLO DE LOS SUBURBIOS LATINOAMERICANOS BAJO NUEVOS PARADIGMAS: REFLEXIONES SOBRE EL EJEMPLO DE JUNDIAÍ, BRASIL'
}

\author{
THE DEVELOPMENT OF LATIN AMERICAN PERIPHERIES UNDER NEW PARADIGMS: \\ REFLECTIONS ON THE EXAMPLE OF JUNDIAÍ, BRAZIL'
}

\section{Patricia Rodrigues Samora²}

RESUMEN

\begin{abstract}
Los asentamientos precarios se han vuelto cada vez más tema de discusión y experimentación en la Arquitectura y Urbanismo, y Latinoamérica alberga intervenciones notables en este ámbito. La mejoría de estos ambientes sigue siendo el principal reto de las ciudades de la región, las que demandan que la arquitectura contemporánea contribuya a partir del rescate de su función social, sin la cual conceptos como sustentabilidad y resiliencia pierden su significado. Este trabajo presenta el caso de la ciudad de Jundiaí, Brasil: un ejemplo del entrelazamiento entre la política habitacional y urbana que se apoya en nuevos instrumentos legales y financiación pública para urbanizar cuatro áreas informales en la ciudad. El caso ilustra cómo estos hitos de la política local le permitieron a la urbe ampliar su escala de operación, aunque a menudo, no se hayan obtenido resultados singulares. Sin embargo, este entrelazado crea una oportunidad para que la Arquitectura amplíe su relevancia social, volviéndose así plenamente sostenible y resiliente.
\end{abstract}

Palabras clave: asentamientos precarios, mejoramiento urbano, diseño urbano, estrategias urbanas, conjuntos habitacionales de alta densidad.

\section{ABSTRACT}

Informal settlements have increasingly become a topic of discussion and experimentation in architecture and urban planning, and Latin America is home to notable interventions in this area. The improvement of these environments is still the main challenge for cities in the region, which require contemporary architecture to contribute by reviving its social function, without which concepts like sustainability and resilience lose their meaning. This article presents the case of Jundiaí, Brazil: an example of the connection between housing and urban policies supported by new legal instruments and public financing to upgrade four slum areas. The case illustrates how these local political milestones helped the city to increase the scale of its intervention, although the results are often unremarkable. However, the connection of different policies creates an opportunity for architecture to broaden its social impact, thus becoming fully sustainable and resilient.

Keywords: informal settlements, slum upgrading, urban design, urban strategies, high-density housing complexes.

Artículo recibido el 21 de agosto de 2018 y aceptado el 4 de diciembre de 2018 DOI: https://doi.org/10.22320/07196466.2018.36.054

[1] Agradecemos al Consejo Nacional del Desarrollo Científico y Tecnológico Brasileño (CNPq) por la beca de Iniciación Científica durante los años 2017 y 2018 para la estudiante de Arquitectura y Urbanismo Sarah Monteferrante, que colaboró con la colecta de datos y elaboración de mapas y figuras que ilustran este artículo.

[2] Docente e investigadora en el Programa de Posgrado en Arquitectura y Urbanismo, Pontifícia Universidade Católica de Campinas, Campinas, Brasil. patricia.samora@puc-campinas.edu.br 


\section{INTRODUCCIÓN}

Las ciudades son sistemas complejos, pero incompletos (Sassen, 2014). En este constante hacerse y deshacerse, las intervenciones en su ambiente construido tienen impactos profundos en diferentes ámbitos. Sin embargo, aunque busquen soluciones arquitectónicas sostenibles, ancladas en nuevos materiales y técnicas, los proyectos de renovación urbana pueden promover efectos adversos, tales como la expulsión de la población local o la alteración en los modos de vida tradicionales.

Las intervenciones en asentamientos precarios constituyen una parte importante de las experiencias de renovación. Los proyectos de urbanización se han vuelto uno de los instrumentos para lograr los Objetivos del Desarrollo Sostenible (ONU Hábitat, 2015; Comisión Económica para América Latina y El Caribe, 2017), los cuales son reconocidos como estrategias para enfrentar las necesidades ambientales y de vivienda de las ciudades y garantizar la permanencia de los habitantes en los barrios que ellos construyeron. Antes, tema predominante en la política de vivienda, el entendimiento de que la complejidad de los asentamientos precarios demanda un compromiso político, que debe ir más allá de la agenda tradicional de las políticas habitacionales (Van Lindert, 2016), provoca y reta a la arquitectura contemporánea. Son campos de ensayo provechosos para soluciones que enfrentan uno de los desafíos contemporáneos más grandes: la promoción de ciudades más justas y equilibradas para todos.

Desde 2007, Brasil amplió los recursos federales para la urbanización de los asentamientos precarios con el Programa de Aceleração do Crescimento - Urbanização de Assentamentos Precários (PAC-UAP). Este texto presenta uno de los casos de estudio de la investigación titulada "A apropriação das ZEIS pelos projetos do PAC Urbanização de Assentamentos Precários e os impactos na qualidade do ambiente construido", que analizó cómo el entrelazamiento entre la política de vivienda y urbana en Brasil, llevado a cabo después de 2003, impactó en la adopción de soluciones urbanísticas y arquitectónicas para desarrollar barrios precarios. Se busca, desde este 
enfoque, comparar los conceptos de sustentabilidad y resiliencia, que han dirigido cada vez más la teoría arquitectónica y urbana contemporánea, a través del ejemplo de Jundiai ${ }^{3}$, ciudad que adoptó el instrumento de las Zonas Especiales de Interés Social (ZEIS) en su política urbana y fue beneficiada por recursos federales del PAC-UAP, entre los años 2007 y 2017. Sumado a ello, se pretende presentar los elementos que condujeron a la adopción de distintas soluciones urbanísticas y de vivienda en la ciudad, entre los cuales la directriz de la sustentabilidad fue determinante en la calidad del hábitat resultante.

La diversidad de soluciones y los problemas verificados son indicativos del papel del diálogo entre las instancias políticas y técnicas que la arquitectura debe asumir progresivamente para producir espacios resilientes y sostenibles a partir del rescate de su función social.

\section{MÉTODO}

Para conocer el alcance del PAC-UAP en el Estado de Sao Paulo, se realizó un inventario total de las 150 intervenciones y un $25 \%$ de estas fueron caracterizadas. De este universo, fueron seleccionadas tres ciudades que utilizaron ZEIS en sus planes directores - aquí se encuentra el caso de Jundiaí. Se llevaron a cabo visitas a cada una de las cuatro intervenciones del PAC en la ciudad y entrevistas a los agentes responsables por ellas, buscando comprender cómo fueron formuladas las estrategias y directrices incorporadas en cada proyecto. Para este artículo, el caso de Jundiaí fue cotejado con los conceptos de sustentabilidad y resiliencia, destacando la importancia de las ZEIS en el alcance de este paradigma.

\section{RESULTADOS}

\section{Sustentabilidad y resiliencia desde el ángulo del rescate de la función social de la arquitectura}

El siglo XXI trajo consigo la concienciación generalizada de las transformaciones estructurales de la sociedad global. Dentro de este contexto, la arquitectura también ha sufrido impactos en sus fórmulas y, para revelarlos, es necesario profundizar en lo que Montaner y Muxi (2015) sintetizan como "vertiente ética" de la posmodernidad, la cual realizó una crítica humanística de la modernidad a partir de los cambios del paradigma de las nuevas sociedades. Así, el corolario que compone la ética de la arquitectura del siglo XXI comprende la arquitectura como política y deberá relacionarse con los cuatro cambios fundamentales que tienen impacto en la sociedad contemporánea: "[...] derechos humanos, sustentabilidad, diversidad y participación" (ibidem, 17). Aquí, la sustentabilidad se refiere a "[...] una nueva conciencia de los límites del crecimiento, del consumo y de la contaminación" (ibidem, 18).

Otro concepto-tipo antagónico a la sustentabilidad, pero que tiene igual repercusión en la arquitectura y el urbanismo, es el de "globalización". Como la ciudad es el locus del capital financiero global, pudiendo explotarse y transformarse con la única finalidad de servir a los intereses de la acumulación, no es suficiente que la arquitectura sea "verde", limitándose a apostar en nuevas tecnologías y en el "diseño resiliente" para que resulte también sostenible. El significado ético de la sustentabilidad en la arquitectura demanda recuperar la función social intrínseca en ella, sirviendo para las necesidades más apremiantes de las ciudades desiguales contemporáneas.

Entre aquellas, el tema del acceso a la vivienda adecuada es una de las prioridades. En el mundo de las megaciudades, el 95\% del aumento poblacional hasta 2050 sucederá en los países en desarrollo (Davis, 2006) y, si no se modifica en algo la dinámica actual, la tendencia es que este crecimiento ocurra en ambientes urbanos precarios e insalubres, ya que las ciudades contemporáneas ya albergan casi 900 millones de personas en asentamientos precarios (ONU Hábitat, 2015), sin infraestructura básica y sujetos a riesgos.

El riesgo y la vulnerabilidad se asocian al concepto de resiliencia urbana que, según la concepción actual, es la capacidad que tienen las ciudades de enfrentar choques y estrés derivados de su condición urbana contemporánea (Rockefeller Foundation, 2014). De este modo, las ciudades latinoamericanas necesitan encarar al mismo tiempo su déficit de infraestructura urbana, el cual resulta del rápido proceso de urbanización de la región, así como lidiar con las incertidumbres del cambio climático que amenazan, sobre todo, a las poblaciones socialmente más vulnerables, habitantes de los lugares menos aptos y seguros.

En Latinoamérica se han desarrollado importantes experiencias de urbanización de asentamientos precarios (Zuquim y Sánchez-Mazo, 2017, que son ejemplos del "revival de las políticas habitacionales en el Sur" (Turok, 2016). Estas se enfocan a menudo en aspectos más amplios que la mera oferta de nuevas unidades habita-

[3] Se investigaron todos los proyectos financiados por el Programa de Aceleração do Crescimento en la modalidad Urbanização de Assentamentos Precários (PAC UAP) en el Estado de São Paulo, buscando comprender cuál es la naturaleza de los proyectos y en qué medida el instrumento de las ZEIS contribuyó a la adopción de soluciones más adecuadas a las necesidades del hábita contemporáneo. El análisis de la política urbana en esta investigación se enfocó el papel de las Zonas Especiales de Interés Social (ZEIS), un instrumento de planeamiento y zonificación que permite tanto legalizar áreas de génesis informal, como delimitar vacíos urbanos para viviendas de interés social. El presente artículo se focaliza en el caso de estudio de Jundiaí, específicamente en la variedad de soluciones encontradas en la ciudad. 
cionales, contribuyendo a las estrategias de desarrollo de las ciudades, tales como un mejor aprovechamiento del suelo urbano o la conectividad y diversidad como objetivos centrales, ampliando así el "premio urbano" (ibidem) para todos.

Las acciones pioneras proponían enfrentar el problema a partir de las estrategias de la autopromoción de la vivienda presentes en las ciudades, valorando el saber de los habitantes (Turner y Fichter,1972) y dotando de infraestructura básica a estos barrios (Van Lindert, 2016). Los experimentos impactaron el debate sobre la vivienda social en Latinoamérica, tanto en el ámbito de las estrategias políticas impulsadas por agencias internacionales de financiamiento y por las reivindicaciones del movimiento social, como en el campo de la arquitectura, que también se transforma al lidiar con este reto.

En las últimas cuatro décadas, la agenda de la política habitacional pasó por transformaciones importantes que acompañaron la propia maduración democrática de la región, en las que las ciudades ganaron más poder y la participación del ciudadano se amplió (ibidem). Al mismo tiempo y principalmente a partir de las privatizaciones de los parques habitacionales públicos en Europa, el avance del neoliberalismo sobre las políticas habitacionales se volvió evidente (Fishman, 2018). El proceso que transformó la vivienda en commodity ha impactado a los pobres en todo el mundo y, al encontrarse con esta realidad, la arquitectura es incitada a que rescate su función social, en la cual la participación del usuario es tan esencial como la adopción de estrategias procesales de proyecto recuperadas de ejemplos como el PREVI (Proyecto Experimental de Vivienda) en Perú en los 60-70 (Barros y Pina, 2012; Fishman, 2018) y actualizadas por nuevas oficinas de arquitectura.

En Brasil, la agenda de la "Reforma Urbana" impulsó cambios hasta en la Constitución Federal del período de la redemocratización, que tuvo que incorporar los retos de la integración de los ambientes informales a la ciudad consolidada, donde vive la mayoría de los pobres urbanos.

\section{Política habitacional y urbana reciente en Brasil}

La experiencia brasileña amplió su alcance y escala a partir de dos hitos: la aprobación del Estatuto de las Ciudades (2001) y la institución de la Política Nacional de Vivienda en 2004 (Antonucci et al., 2017).

El citado Estatuto es la Ley Federal 10.257/01, cuyo punto céntrico es el refuerzo del papel municipal -presente en los artículos 182 y 183 de la Constitución Federal de 1988- referente a la planificación y ejecución de la política habitacional y urbana, que busca superar el pasivo histórico de las ciudades brasileñas en cuanto al riesgo e insalubridad del hábitat. Entre sus herramientas de planificación y gestión, la definición de las Zonas Especiales de Interés Social (ZEIS) permite que los municipios enfrenten sus problemas habitacionales directamente y tienen dos modalidades: regularización e inducción. La primera permite regularizar cualquier asentamiento urbano de interés social según parámetros urbanísticos propios; se aplica a la mayoría de los asentamientos precarios y hace posible la regularización urbanística y de tenencia de estos locales. Las ZEIS de inducción, o "ZEIS de los vacíos", apoyan la política de tierras local, al delimitar los terrenos urbanos aptos para la implantación de vivienda social, ya sea de promoción pública o de mercado.

El segundo hito fue la Política Nacional de Habitación de 2004, que establece condiciones para que los municipios accedan a los recursos de la federación para urbanizar y construir nuevas viviendas. Así, se lanzaron programas como el PAC -Urbanização de Assentamentos Precários, de 2007, y el Programa Minha Casa Minha Vida, de 2009, que financia nuevas unidades habitacionales para los habitantes urbanos pobres.

La dificultad de expropiación de áreas para la construcción de viviendas ha impactado los proyectos de urbanización. Ellos terminan apenas removiendo las viviendas bajo riesgo inminente, manteniendo muchas veces sectores muy densos o precarios dentro del asentamiento urbanizado (Zuquim, 2012). De esto surge la importancia de los instrumentos como las ZEIS que, en un contexto de fuerte valoración de los inmuebles, reservan en la ciudad áreas vacías buscando atender el déficit habitacional de la población más vulnerable. EI principal obstáculo para que las políticas habitacionales municipales produzcan espacios con mayor calidad todavía es el acceso a terrenos urbanizados (Denaldi y Bruno Filho, 2013; Fonseca, Fernandes y Tavares, 2013). Según Fernandes (2016), un avance del Estatuto de las Ciudades "fue la creación de Zonas Especiales de Interés Social (ZEIS) "Ilenas", o sea, correspondientes a los asentamientos informales existentes". Sin embargo, pocos municipios propusieron opciones de vivienda social en las áreas centrales; la enorme mayoría no reservó tierras para producción habitacional de interés social (las ZEIS "vacías"). Este hecho produce un contraste con el diagnóstico de las ciudades brasileñas realizado por el mismo autor, en las cuales

\footnotetext{
"[...] hay enormes existencias de tierras urbanas provistas de servicios públicos pero mantenidas vacías por sus propietarios, correspondiendo en algunos casos al $20 \%$ de la red urbana del municipio/región metropolitana), así como de construcciones vacías, abandonadas y/o sub-utilizadas (que llega a 5,5 millones de unidades [...]." (ibidem, 32-33)
}

Idealmente, las ZEIS crean condiciones para rehabilitar a los barrios informales bajo nuevos paradigmas de calidad. La regularización de las áreas informales con parámetros urbanísticos propios para cada caso, le brinda a las ZEIS el papel estratégico de ampliar la flexibilidad de soluciones y la diversidad de modelos para atender a un derecho humano central: el derecho a la vivienda adecuada. Cuando se reservan áreas urbanas vacías o infrautilizadas para fines habitacionales, permitiendo incluso la mezcla de usos, si 
así se define en el Plan Directivo municipal, las ZEIS de inducción democratizan el uso del suelo urbano ya dotado de infraestructura y servicios, actuando en la transformación de ciudades más justas e inclusivas, por lo tanto, más sostenibles y resilientes.

Asimismo, otro aspecto observado es que la rigidez del programa Minha Casa Minha Vida contribuye a la falta de calidad de la producción habitacional, aunque cuente con subsidios altos, ya que se apoya únicamente en el financiamiento de la vivienda. El recurso no se aplica a la construcción de viviendas en terrenos en proceso de legalización, como es el caso de muchos asentamientos precarios, inclusive los de Jundiaí. De este modo, muchas ciudades optaron por trasladar a los habitantes hacia emprendimientos situados en terrenos distantes del asentamiento original con el propósito de volver viables las obras de infraestructura urbana para la porción remanente que se iba a urbanizar. Este fue el arreglo posible entre el repaso de los recursos financieros de la federación, la acción municipal y la coyuntura con el mercado de tierras, que alimentó el proceso especulativo y la segregación (Rolnik et al., 2015; Carvalho, Shimbo y Rufino, 2015).

\section{El caso de Jundiaí, Brasil}

El entrelazamiento de la política habitacional y urbana ha sido uno de los retos planteados a los municipios brasileños. El caso de Jundiaí ejemplifica las estrategias adoptadas en este escenario, las cuales se ilustran a través de cuatro intervenciones en ZEIS de regularización: Vila Ana, Parque Centenário, Jardim Novo Horizonte y Jardim São Camilo.

Como otras ciudades medianas brasileñas, la vitalidad económica y el liderazgo regional de Jundiaí no se reflejan en un espacio urbano con calidad para todos, y muchos de sus habitantes residen en asentamientos informales. La precariedad se manifiesta de forma diversa, así como la situación urbana de cada localidad es única, lo que forzó la búsqueda de respuestas distintas (Jimenez, 2018). Jundiaí amplió la escala de intervención en los barrios a partir de la estructura legal del Estatuto de las Ciudades, que le da al municipio el protagonismo de su política urbana.

En lo que sigue se presenta una breve descripción de cada una de las cuatro intervenciones, a partir de un cuadro general (Tabla 1) de las distintas formas urbanas de precariedad en ZEIS. Es importante mencionar que esta variedad de soluciones son, de alguna manera, también otorgadas por las pautas del PAC UAP, que permitieron a los municipios la libertad de formular planes de acción para cada área a mejorar, sin imponer modelos urbanos. Sin embargo, si los resultados no presentan arquitectura de calidad singular, los cuatro casos brindan soluciones de variadas densidades y tipos, demostrando el campo de experimentación posible que se desea resaltar en este artículo.

Vila Ana

Vila Ana se localiza en la porción sur del centro del municipio de Jundiaí, cerca de la región central de la ciudad, entre la vía Nove de Julho, eje comercial local, y la Autopista Anhanguera. El núcleo estaba compuesto -antes de ser intervenido-por 5 cuadras con 159 viviendas precarias. La alta densidad de ocupación (según la tabla 1) y la precariedad requirieron la remoción de todas las viviendas originales (Figura 1). A pesar de continuar siendo predominantemente residencial, el local está bien equipado de equipo urbanos. La solución del arquitecto Pier Paolo Pizzolato implantó 22 bloques con 144 departamentos, formando 5 manzanas aisladas y delimitadas por las vías, con edificios que se insertan de forma discreta en el paisaje (Figura 2).

\begin{tabular}{|c|c|c|c|c|}
\hline Asentamiento & Vila Ana & Parque Centenário & São Camilo & Novo Horizonte \\
\hline Población estimada (personas) & 477 & 444 & 4.879 & 8.891 \\
\hline Área (ha) & 0,87 & 3,10 & 14,40 & 42 \\
\hline $\begin{array}{l}\text { Población estimada después de la intervención } \\
\text { (personas) }\end{array}$ & 452 & 355 & 3675 & 8025 \\
\hline Viviendas después de la intervención & 144 & 113 & 1221 & 2897 \\
\hline Densidad anterior a la intervención (hab/ha) & 513,87 & 150,14 & 338,2 & 211,69 \\
\hline Densidad después de la intervención (hab/ha) & 519,72 & 114,45 & 255,22 & 191,06 \\
\hline
\end{tabular}



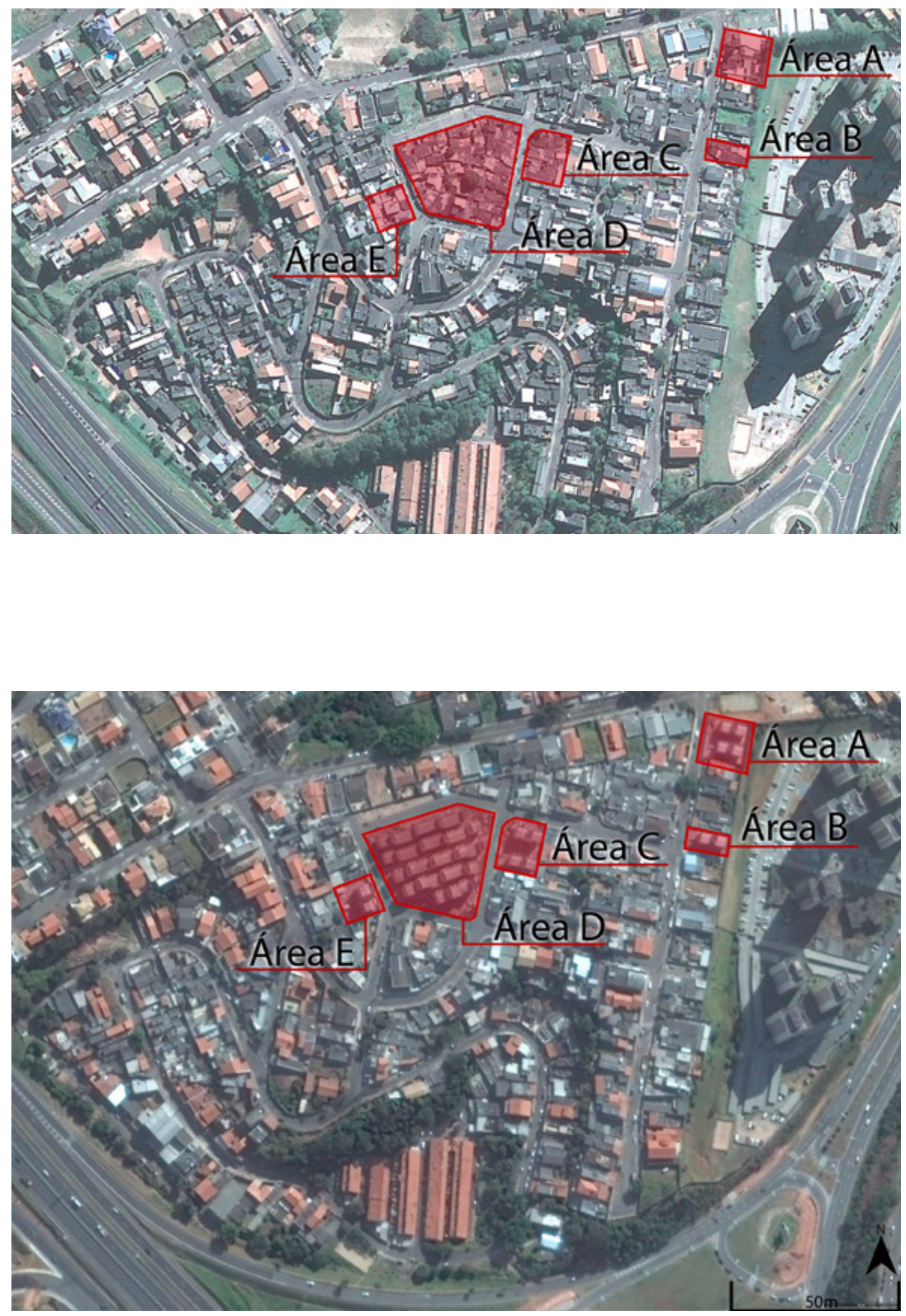

Figura 1 Vila Ana. Tejido urbano antes y después de la intervención. Fuente: Elaborado por Sarah Monteferrante sobre imágenes Google tomadas en 2009 y 2017. 

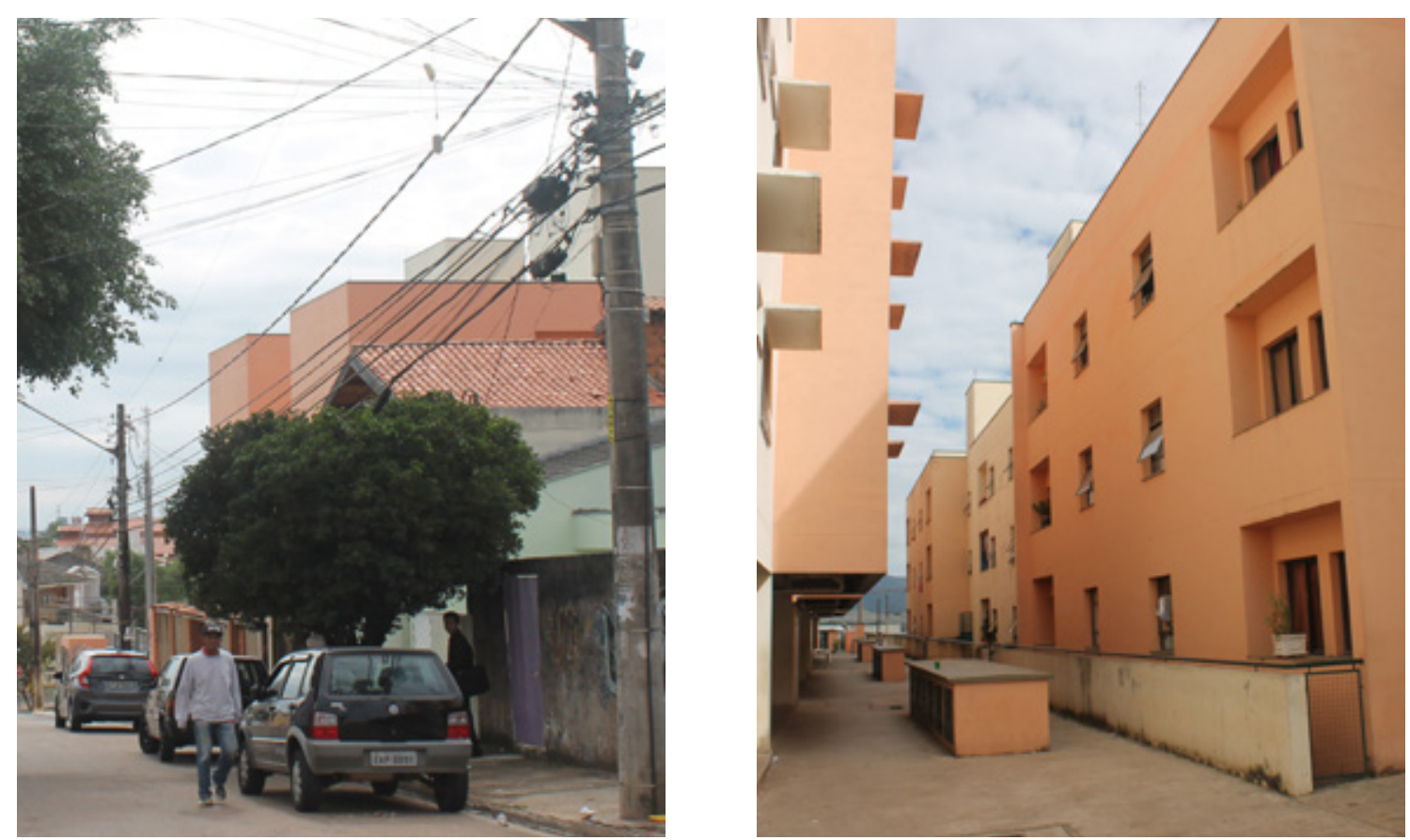

Figura 2 Vila Ana. Inserción de los bloques en el tejido urbano (izquierda) y detalle de los bloques de departamentos (derecha). Fuente: Fotografías de Patrícia Samora, 2017.

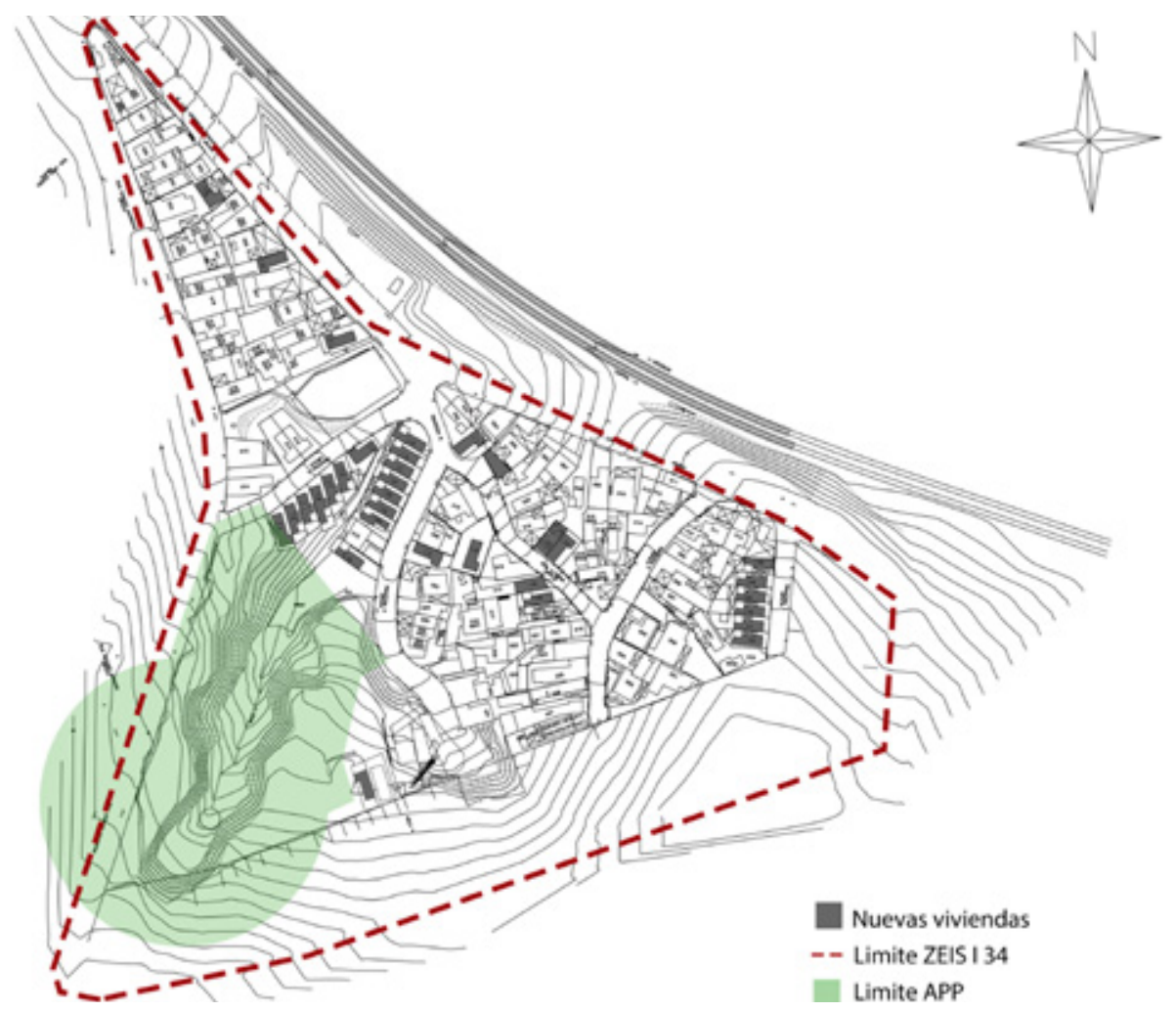

Figura 3 Parque Centenário. Plano urbanístico con ubicación de las nuevas viviendas. Fuente: Elaborado por Sarah Monteferrante a partir de datos de la Prefeitura Municipal de Jundial. 


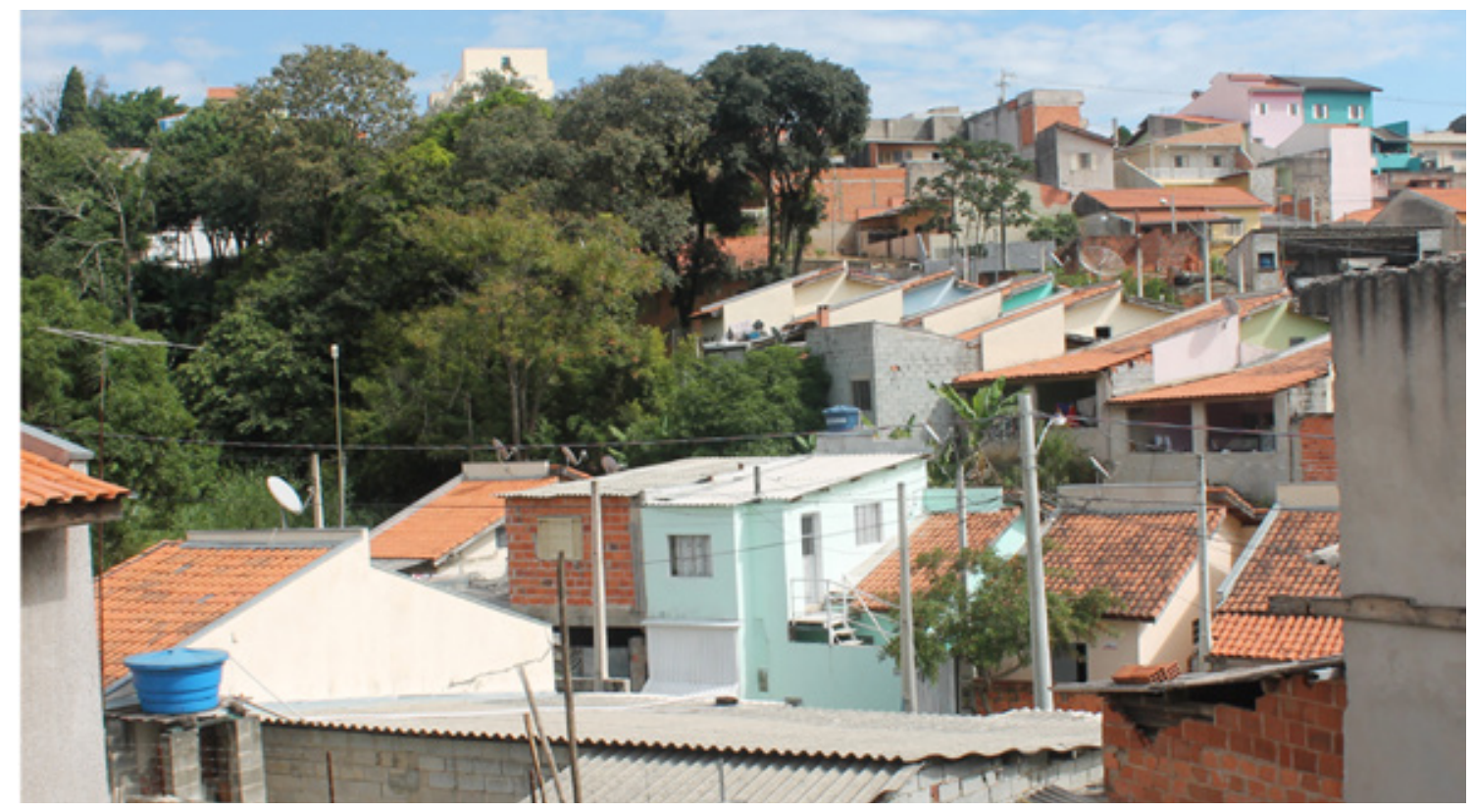

Figura 4 Parque Centenário. Casas en hilera junto a la APP, ya transformadas por los residentes. Foto: Patrícia Samora, 2017.

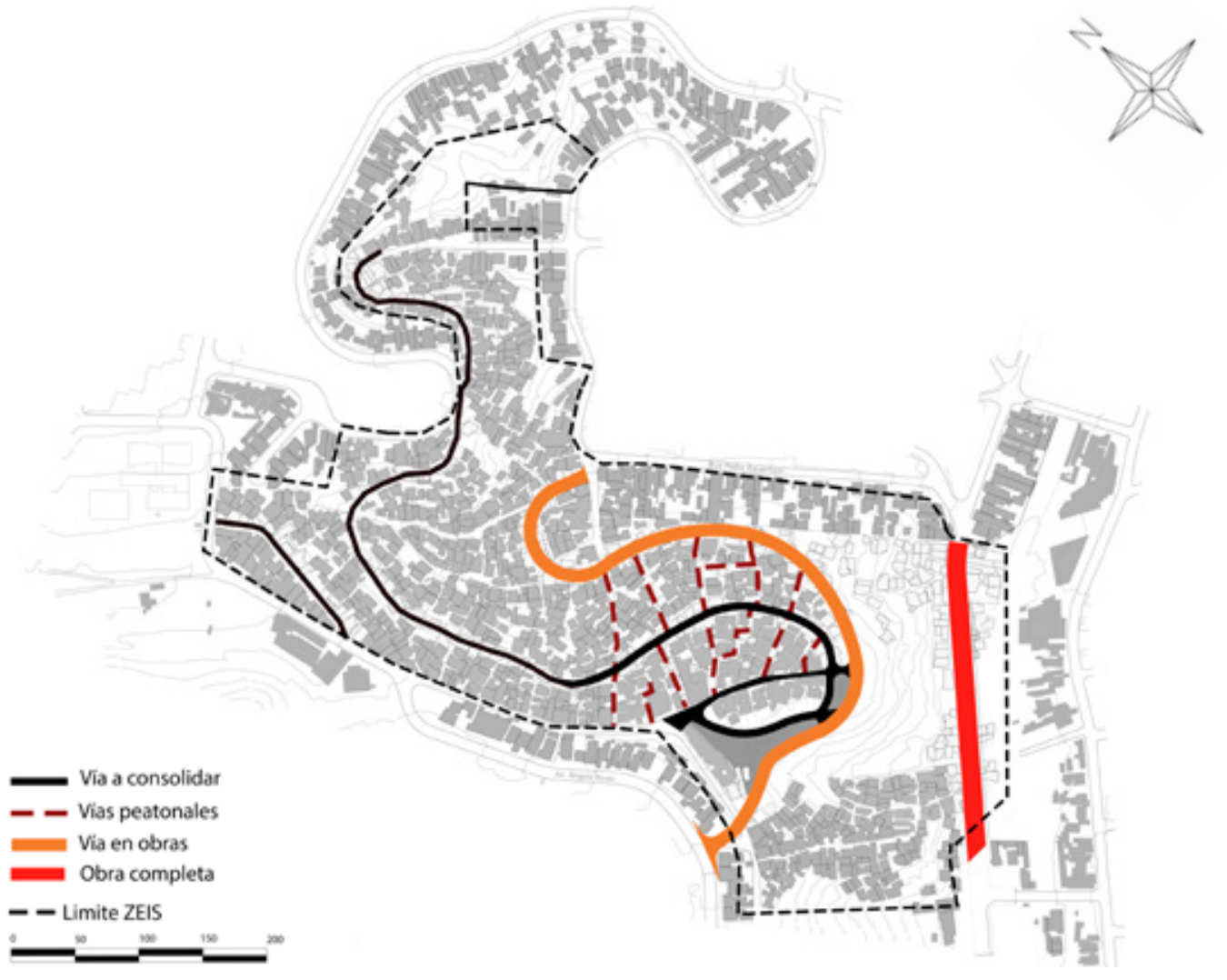

Figura 5 Plano urbanístico para Jardim São Camilo. Fuente: Elaborado por Sarah Monteferrante a partir de datos de la Prefeitura Municipal de Jundiaí. 


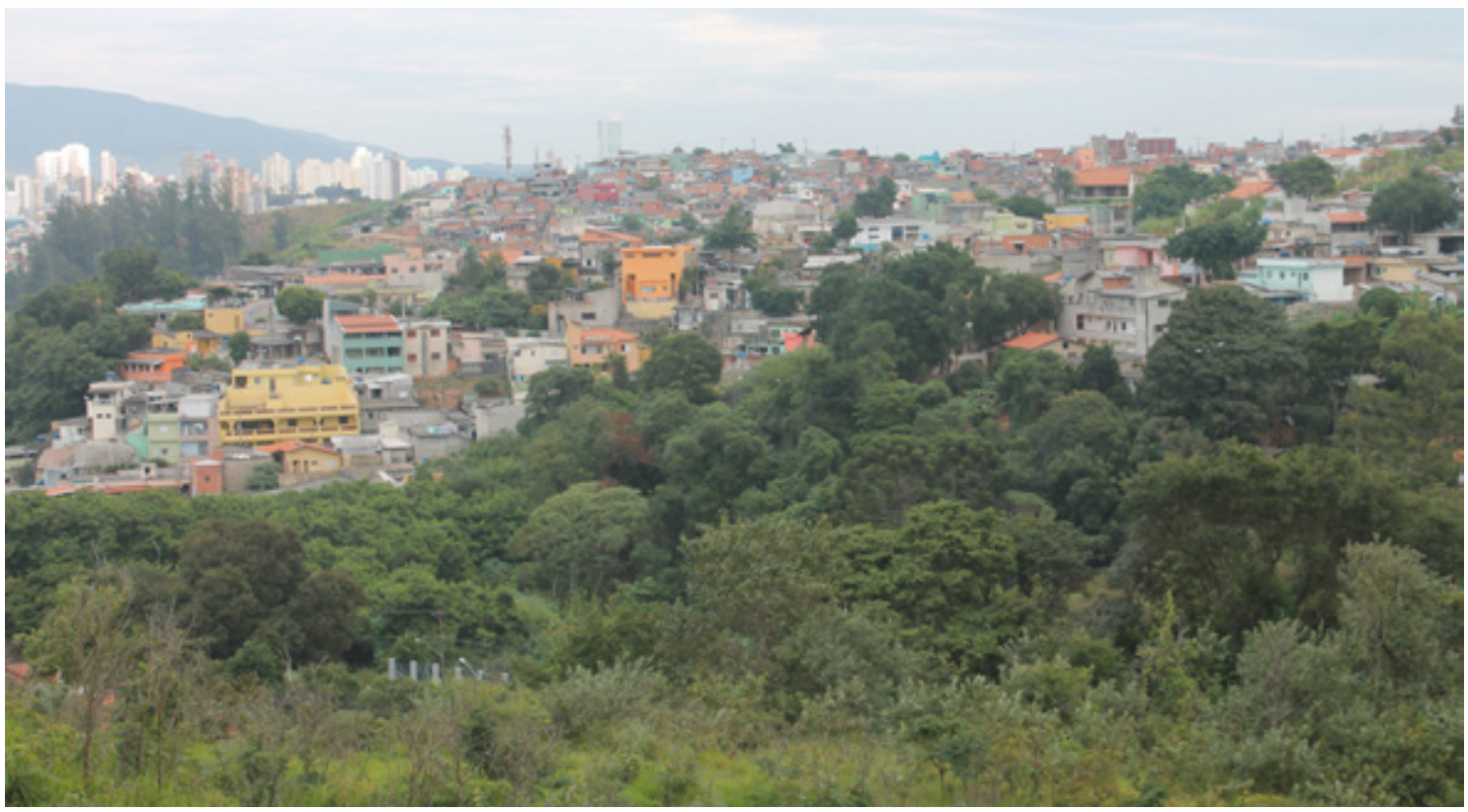

Figura 6 Jardim São Camilo. Complejidad de la topografía y alta densidad constructiva. Fuente: Fotografía de Patrícia Samora, 2017

\section{Parque Centenário}

El asentamiento tenía 148 hogares antes de la intervención, con vías dotadas de saneamiento básico, pero sin pavimentación. En el sitio hay una fuente, área protegida por ley (Área de Preservación Permanente - APP). Los caminos precarios fueron consolidados mejorando la integración con los alrededores, y las casas bajo riesgo, aquellas situadas en áreas con restricciones ambientales y otras ubicadas junto al cauce ferroviario, fueron removidas (Figura 3). La solución propuesta mantuvo el tejido de baja densidad predominante, con casas en hileras que no impactaron el paisaje.

Fueron implantadas 33 nuevas viviendas, oponiéndose a las 68 removidas (Tabla 1) y se adoptó un tipo habitacional evolutivo que los habitantes podían ampliar, proyecto del ingeniero Jaime Chiguero Daedo. La intervención fue simple, sin arquitectura singular (Figura 4), tal vez debido a la condición de la periferia y de baja complejidad del asentamiento, lo que difiere de la situación urbana de Vila Ana, por ejemplo.

\section{Jardim São Camilo}

Jardim São Camilo es un área densa localizada en una zona urbana consolidada, cerca del centro y con buena accesibilidad. Alberga 1.618 viviendas con 4.879 habitantes. El sitio se caracteriza por la pendiente empinada ocupada por viviendas de bajo riesgo. Fueron removidas 397 de ellas para mejorar la accesibilidad y crear un parque junto al curso de agua canalizado (Figuras 5 y 6). La vía propuesta por la intervención, la que recorre toda la ladera, aún no ha sido finalizada. Los residentes retirados fueron trasladados a un emprendimiento a $1 \mathrm{~km}$ del sitio original, lo que dio como resultado una disminución de la densidad del asentamiento (Tabla 1).

El nuevo emprendimiento (Figura 7) posee 400 unidades habitacionales de $46 \mathrm{~m}^{2}$, dispuestas en 25 bloques alineados con 4 pisos cada uno. La circulación vertical está dada por escaleras y ascensor, con acceso en galería a los departamentos. El proyecto ignoró la topografía empinada, realizando un gran desplazamiento de tierra para construir los edificios. Como consecuencia, se crearon grandes espacios libres e impermeabilizados entre los dos bloques, para el acceso de vehículos, ya que no se previeron espacios de uso colectivo, exceptuando un centro comunitario. La concepción arquitectónica del emprendimiento fue de la propia alcaldía/ ayuntamiento y desarrollada por el grupo Pentarco Engenharia e Arquitetura.

\section{Jardim Novo Horizonte}

Jardim Novo Horizonte está más alejado del centro consolidado y tiene un tipo urbano peculiar: un asentamiento lineal de $7 \mathrm{~km}$ de longitud cuya forma deriva del proceso de ocupación de un antiguo cauce ferroviario. Los alrededores se caracterizan por la presencia de industrias, por grandes glebas no ocupadas y por nuevos conjuntos de viviendas construidos por el poder público local y estatal, ya que la región es el principal destino de la producción de los programas habitacionales actuales y anteriores. Hay equipos, como escuelas y puestos médicos para atender a los residentes. 


\section{PREPRINT}

AS54

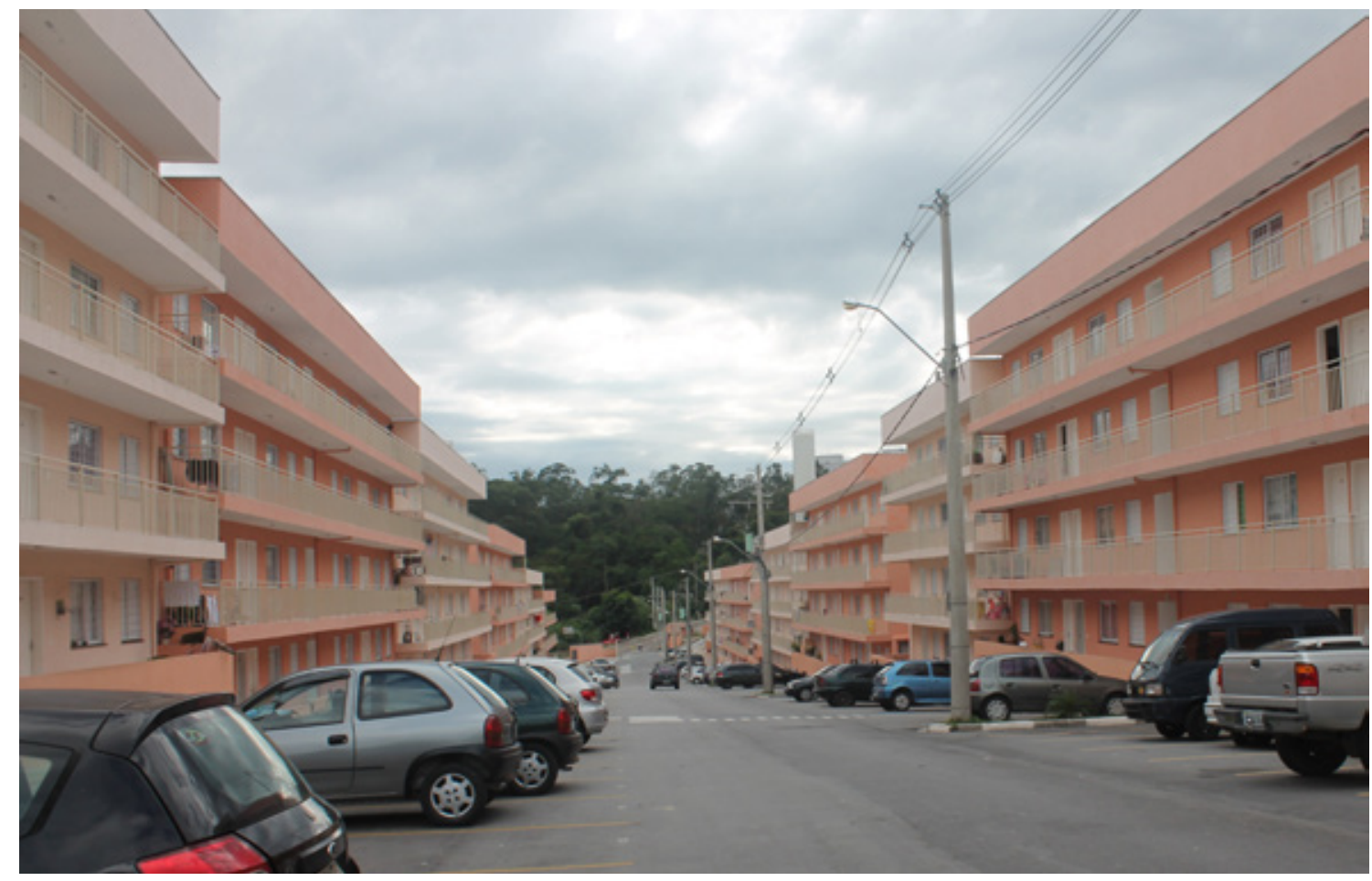

Figura 7 Jardim São Camilo. Nuevos bloques para albergar a los habitantes removidos por la urbanización del asentamiento, que está ubicado tras los árboles. Fuente: Fotografía de Patrícia Samora, 2017.

$\Lambda$

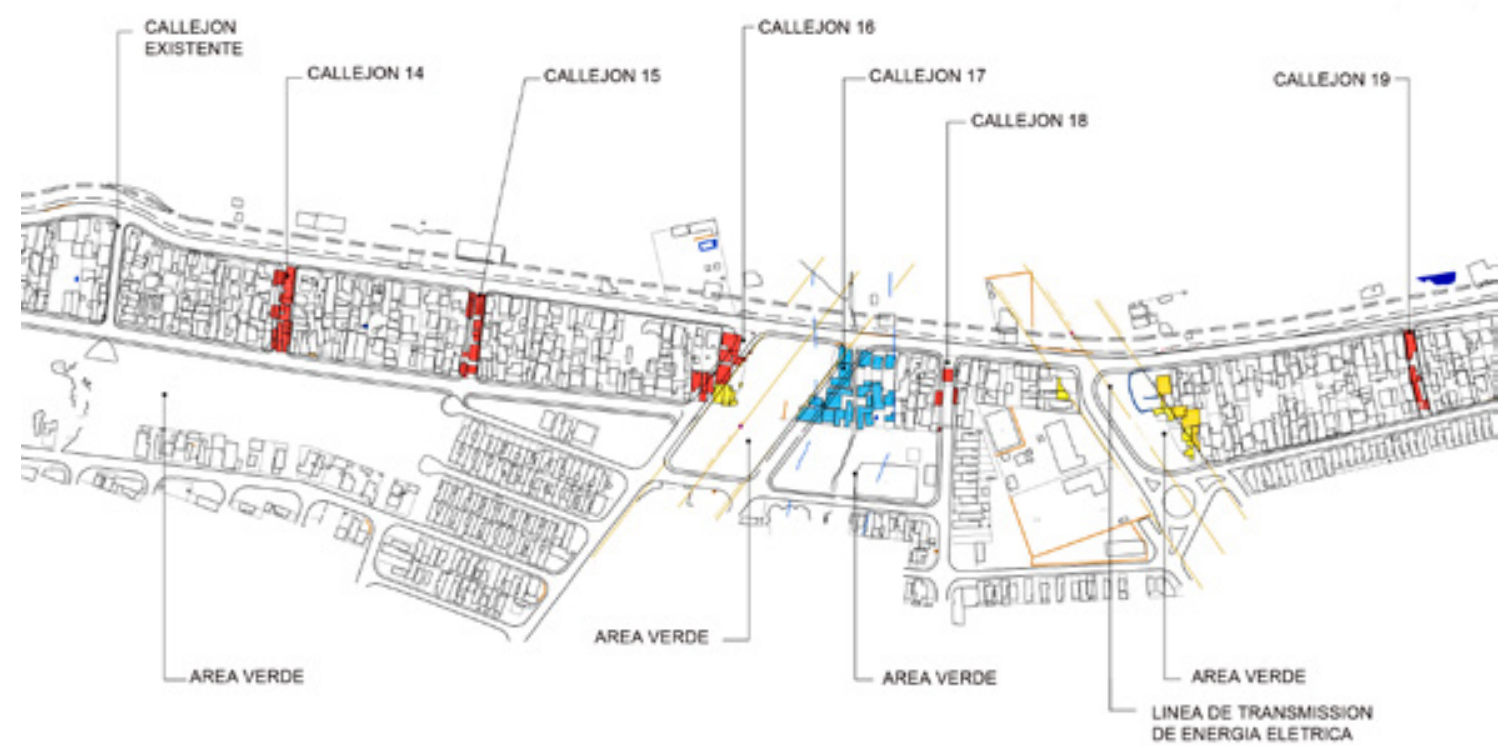

Figura 8 Jardim Novo Horizonte. Nuevos callejones planeados para ampliar la conexión con el entorno. Fuente: Elaborado por Sarah Monteferrante a partir de datos de la Prefeitura Municipal de Jundiaí. 


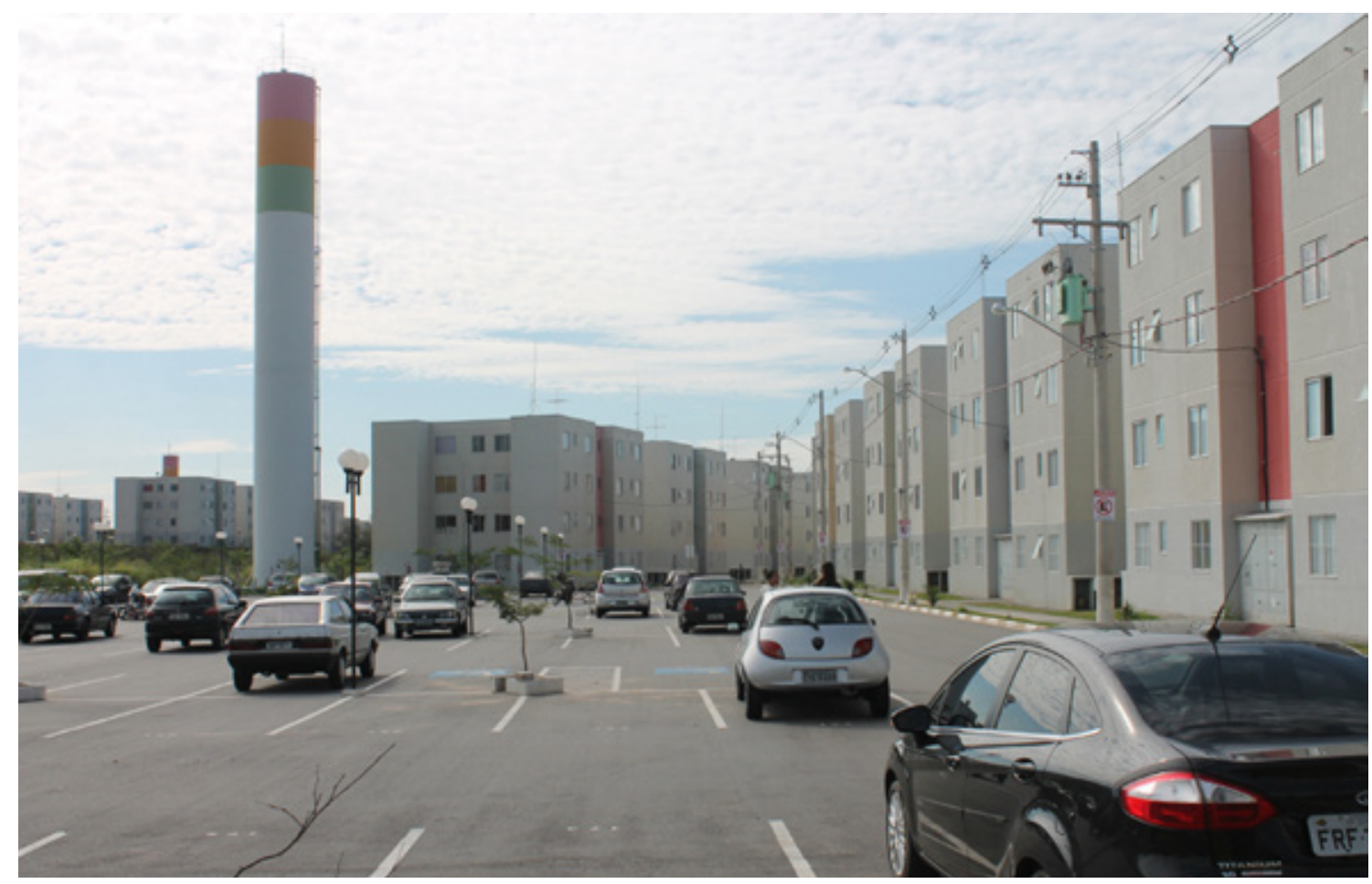

Figura 9 Conjunto de vivienda del Programa Minha Casa Minha Vida, con sus bloques estandarizados, donde los residentes removidos del Jardim Novo Horizonte fueran trasladados. Fuente: Fotografía de Patrícia Samora, 2017.

Antes de la intervención, alrededor de 8.891 personas residían en Jardim Novo Horizonte en 3.112 inmuebles registrados, algunos bastante precarios. La principal necesidad del sitio, además de la regularización agraria y la rehabilitación de las situaciones precarias, es la apertura de conexiones viales transversales (Figura 8). De las 35 vías previstas en el proyecto de la oficina Projeto Paulista, sólo 7 han sido concluidas hasta el momento. Algunos domicilios tampoco se han podido consolidar porque están en áreas de protección permanente por causa de los arroyos presentes en el barrio. El financiamiento de la obra de urbanización recibió recursos del PAC y del Gobierno del Estado.

Un conjunto habitacional cerca del área, con 1.088 unidades (Figura 9), fue el destino de los habitantes removidos. Este se encuentra conformado por bloques estandarizados, con departamentos de dos tipologías, cuyas áreas oscilan entre los 34 y los 38 $\mathrm{m}^{2}$, característicos de la producción en masa del programa Minha Casa Minha Vida. Con la paralización de las obras en Novo Horizonte, el emprendimiento fue ocupado también por habitantes de otras regiones de la ciudad.

\section{CONCLUSIONES}

El análisis de los cuatro casos aquí expuestos reveló algunos retos que aún están vigentes y que tienen un impacto directo en la calidad del ambiente construido. El principal de ellos es el modelo del financiamiento habitacional que, aunque recibe subsidios altos, no permite la construcción de viviendas en los perímetros originales de los asentamientos informales, ya que el banco financiador exige inmuebles legalizados para la producción habitacional. No obstante, la regularización agraria de asentamientos informales fue un proceso retardado, concluido mucho después de haberse finalizado las obras de infraestructura.

En el caso de Vila Ana, los residentes se quedaron en locales provisionales durante la construcción, lo que fue motivo de desgaste. En el Parque Centenário, la solución encontrada les proporcionó a los habitantes un tipo habitacional incompleto, que culminaría tras la ocupación del usuario, pues una vez más, la irregularidad del terreno impidió el acceso a líneas de financiación de la vivienda. La diferencia de la calidad 
arquitectónica entre Vila Ana y Parque Centenario puede indicar que la ubicación más periférica del último ha pesado en la elección de un proyecto que ni siquiera fue desarrollado por un arquitecto (faltándole la calidad de la vivienda incremental como propuesta por la oficina Elemental, por ejemplo); hecho comprobado en otros casos de la investigación no divulgados en este artículo.

Los casos de São Camilo y Jardim Novo Horizonte demuestran cómo las ZEIS de los vacíos se pueden asociar a la urbanización de áreas de alta densidad, donde es necesario remover casas para implantar las redes de infraestructura, crear áreas libres y contener los riesgos geotécnicos. El impacto en la vida del usuario es menor si los residentes pueden permanecer en el barrio de origen, de donde se origina el uso de las ZEIS de vacíos, las que pueden ser utilizadas por la municipalidad como herramienta para reservar locales más cercanos con este propósito. Cuando el área reservada para las ZEIS es superior a $500 \mathrm{~m}^{2}$, ciudades como Sao Paulo permiten que el comercio y el servicio local sean incluidos en el plano de desarrollo local, mejorando la urbanidad resultante. Sin embargo, el programa Minha Casa Minha Vida no financia otros inmuebles además de los habitacionales y ha recibido críticas pues, con esta limitación, barrios dormitorios, de gran escala y estandarizados, acaban por ser el destino de los habitantes removidos de las favelas. Es decir, aunque la política urbana permite una reformulación integral de los barrios bajo los nuevos paradigmas ambientales y de calidad urbana, la política de financiación de vivienda crea significativas limitantes para lograr tal objetivo. Sin embargo, si las reglas de los programas de financiamiento de vivienda disponibles no hacen posible planificar e implementar inmuebles con usos distintos del de vivienda, el municipio debe afrontar esta cuestión. Esto puede requerir una inversión de recursos municipales adicionales para contemplar todas las necesidades del hábitat, evitando que barrios con miles de viviendas nuevas sean integrados a la ciudad sin los espacios necesarios para la vida urbana integral.

La superación del patrón de baja calidad de las periferias urbanas en el continente depende del compromiso de arquitectos y gestores públicos con la adopción de nuevos modelos y procesos que contemplen el paradigma contemporáneo de la vivienda. En él, la sustentabilidad significa dinamizar el uso del suelo urbano con diversidad de funciones y estructuras, y con bajo impacto ambiental en toda la cadena de las decisiones de proyecto. La resiliencia, a su vez, se traduce en ambientes versátiles, adaptados a la realidad económica, social y cultural de los usuarios.

Jundiaí buscó enfrentar la precariedad del ambiente habitado con soluciones específicas para cada caso, sacando ventajas de instrumentos urbanísticos como las ZEIS. Sin embargo, la calidad obtenida no fue homogénea para todas las familias beneficiadas. La adopción de tipos habitacionales estandarizados, como aquellos destinados a los residentes retirados del Parque Centenario, ejemplifica los retos que aún permanecen en la compleja tarea de mejorar las periferias en el continente.

\section{AGRADECIMIENTOS}

Se agradece al Consejo Nacional del Desarrollo Científico y Tecnológico Brasileño (CNPq) por la beca de Iniciación Científica durante los años 2017 y 2018 para la estudiante de Arquitectura y Urbanismo Sarah Monteferrante, quien colaboró con la colecta de datos y elaboración de mapas y figuras que ilustran este artículo. 


\section{REFERENCIAS BIBLIOGRÁFICAS}

ANTONUCCI, Denise; PRESTES, Márcia Ferreira; SAMORA, Patrícia Rodrigues y ZUQUIM, Maria de Lourdes. Da luta pela moradia à urbanização de assentamentos precários: A política habitacional no Brasil. En: ZUQUIM, Maria de Lourdes y SÁNCHEZ-MAZO, Liliana Maria. Barrios populares Medellín. Favelas São Paulo [en línea]. São Paulo: Faculdade de Arquitetura e Urbanismo, 2017, pp.48-65. [Consultado 18 noviembre 2017]. Disponible en: http://www.favelasaopaulomedellin.fau.usp.br/wpcontent/uploads/2015/07/Barrios-Populares-MedellinFavelas-Sao-Paulo.pdf>.

BARROS, Raquel y PINA, Sílvia. Sinfonia inacabada da habitação coletiva: lições a partir do PREVI para uma arquitetura de possibilidades, Ambiente construido, 2012 vol.12, $n^{\circ} 3$, pp. 7-26.

CARVALHO, Caio Santo Amore de; SHIMBO, Lúcia Zanin y RUFINO, Maria Beatriz Cruz. Minha casa... e a cidade? avaliação do Programa Minha Casa Minha Vida em seis estados brasileiros. Rio de Janeiro: Letra Capital, 2015.

COMISIÓN ECONÓMICA PARA AMÉRICA LATINA Y EL CARIBE. Plan de Acción Regional para la implementación de la Nueva Agenda Urbana en América Latina y el Caribe: 2016-2036 [en línea]. Santiago: Naciones Unidas, 2017. [Consultado 12 agosto 2018]. Disponible en: https:// www.cepal.org/sites/default/files/events/files/par_esp_ c1700844_web1.pdf

DAVIS, Mike. Planeta Favela. São Paulo: Boitempo editorial, 2006.

DENALDI, Rosana y BRUNO FILHO, Fernando. Plano Diretor, Zonas Especiais de Interesse Social e a articulação com o Plano Local de Habitação. En: DENALDI, Rosana (org.). Planejamento Habitacional: Notas sobre a precariedade e terra nos Planos Locais de Habitação. São Paulo: Annablume, 2013, pp. 267-283.

FERNANDES, Edesio. Estatuto de la Ciudad, 15 años después: ¿razón de descrédito o razón de optimismo? En: ROSSBACH, Anaclaudia (org.). Estatuto de la Ciudad: la vieja y la nueva agenda urbana. Un análisis de 15 años de la ley [en línea]. São Paulo: Cities Alliance, 2016. [Consultado 15 agosto 2018]. Disponible en: http:// www.citiesalliance.org/sites/citiesalliance.org/files/ estatuto_da_cidade_15_anos_sitell.PDF

FISHMAN, Robert. The Global Crisis of Affordable Housing: Architecture Versus Neoliberalism, Architectural Design [en línea], jul. 2018, vol. 88, nº 4, pp. 22-29. DOI: 10.1002/ad.2317.

FONSECA, Maria de Lourdes; FERNANDES, Camila Nastari y TAVARES, Helga Canedo. Terra para Habitação de Interesse Social na Região Metropolitana de São Paulo. En: DENALDI, Rosana (org.). Planejamento Habitacional: Notas sobre a precariedade e terra nos Planos Locais de Habitação. São Paulo: Annablume, 2013, pp. 285-307.

JIMENEZ, Flavia Tarricone. Urbanização de assentamentos precários em Jundiaí (SP) 2010-2017: as intervenções dos Programas de Aceleração do Crescimento, Minha Casa
Minha Vida e o papel da Fundação Municipal de Ação Social. Tesis de maestria inédita, Pontifícia Universidade Católica de Campinas, 2018.

MONTANER, Josep Maria y MUXI, Zaida. Arquitetura e Política: Ensaios para mundos alternativos. São Paulo: G. Gili Ltda., 2015.

ONU HABITAT. Transformar nuestro mundo: La agenda 2030 para el Desarrollo Sostenible [en línea]. Quito: Naciones Unidas, 2015. [Consultado 15 agosto 2018]. Disponible en: http://undocs.org/es/A/RES/70/1

ROCKEFELLER FOUNDATION. How to Build a Resilient City: The City Resilience Framework [en línea]. The Rockefeller Foudation, 2014. [Consultado 15 agosto 2018]. Disponible en: https://assets.rockefellerfoundation.org/ app/uploads/20140917211908/Remarks-Nancy-Kete. pdf

ROLNIK, Raquel; PEREIRA, Alvaro Luis dos Santos; MOREIRA, Fernanda Accioly; ROYER, Luciana de Oliveira IACOVINI, Rodrigo Faria Gonçalves y NISIDA, Vitor Coelho. Programa Minha Casa Minha Vida nas regiões metropolitanas de São Paulo e Campinas: aspectos socioespaciais e segregação, Cadernos Metrópole [en línea], 2015, vol. 17, n³3, pp.127-154. DOI: 10.1590/22369996.2015-3306.

SASSEN, Saskia. The city: Today's frontier zone, Glocalism: Journal of Culture, Politics and Innovation [en línea], 2014 n³, pp.1-4. DOI: 10.12893/gjcpi.2014.3.1.

TURNER, John y FICHTER, Robert. Freedon to Build. New York: Macmillan Publishers, 1972

TUROK, Ivan. Housing and the urban premium, Habitat International [en línea], 2016, vol. 54, pp. 234-240. DOI: 10.1016/j.habitatint.2015.11.019.

VAN LINDERT, Paul. Rethinking urban development in Latin America: A review of changing paradigms and policies, Habitat International [en línea], 2016, vol. 54, pp. 253-264. DOI: 10.1016/j.habitatint.2015.11.017.

ZUQUIM, Maria de Lourdes. Urbanização de assentamentos precários no município de São Paulo: quem ganha e quem perde? En: II Encontro da Associação Nacional de Pesquisa e Pós-graduação em Arquitetura e Urbanismo: Teorias e práticas da Arquitetura na cidade contemporânea: Complexidade, mobilidade, memória e sustentabilidade. Natal, 18-21 de setiembre de 2012 . Natal: EDUFRN, 2012, vol. 1, pp. 245-255.

ZUQUIM, Maria de Lourdes y SÁNCHEZ-MAZO, Liliana Maria. Barrios populares Medellín. Favelas São Paulo [en línea]. São Paulo: Faculdade de Arquitetura e Urbanismo, 2017. [Consultado 15 agosto 2018]. Disponible en: http:// www.favelasaopaulomedellin.fau.usp.br/wp-content/ uploads/2015/07/Barrios-Populares-Medellin-FavelasSao-Paulo.pdf 